\title{
Monofilament whisker-based mobile robot navigation
}

\author{
Rishabh Shukla ${ }^{1}$, Prasanna Kumar Routray ${ }^{1}$, Kshitij Tiwari ${ }^{2}$, Steven M. LaValle ${ }^{2}$, M. Manivannan ${ }^{1}$
}

\begin{abstract}
Rodents and pinnipeds use their tactile sense as the primary modes for localization and foraging. This abstract presents a rodent whisker-inspired tactile sensor for mobile robot navigation fabricated using the Semmes-Weinstein Monofilament (SWMF). The monofilament is lightweight, readily available, and generates reproducible buckling stresses. The sensor deflection characteristic has three distinct regions of operation that help detect buckling. The sensor's small size, $360^{\circ}$ tactile view, and force sensing capability could make it suitable for narrow space navigation.
\end{abstract}

\section{INTRODUCTION}

Tactile sensing depends on the sense of touch to interact with the world. In the case of rodents and pinnipeds, whiskers (vibrissae) present in their snout amplify vibrations and give them specialized tactile sense for navigating complex environments [1]. Rodents often use whiskers as a proximity sensor and, in some cases, detect the airflow. In contrast, pinnipeds predominantly use whiskers to detect perturbations in the flow around them. Although whiskers are the primary navigation sense in many animals, their application in mobile robots needs more investigation [2], [3].

\section{METHOD}

The prototype uses an SWMF 5.07 (an engineered material designed to buckle at $10 \pm 1 \mathrm{~g}$ load [4], [5]) as the whisker. Fig. 1(A) shows the schematic of a sensor module. A rubber diaphragm supports an SWMF whisker, with a cylindrical neodymium magnet attached at the base. A 3D hall-effect transducer below the magnet acts as the whisker follicle. The SWMF's diameter is $0.43 \mathrm{~mm}$, and the length is $36.50 \mathrm{~mm}$, and the magnet's diameter is $2.00 \mathrm{~mm}$, and the length is $3.00 \mathrm{~mm}$. The hall-effect sensor is $2.96 \mathrm{~mm} \times 2.32$ $\mathrm{mm} \times 2.50 \mathrm{~mm}$, and the enclosure is $17 \mathrm{~mm} \times 12 \mathrm{~mm} \times 10$ $\mathrm{mm}$. The hall-effect sensor's sensitivity and latency are 7.7 $\mathrm{LSB} / \mathrm{mT}$ and $0.125 \mathrm{~ms}$, respectively. The magnet's deflection range is $\pm 5 \mathrm{~mm}$, which translates to $\pm 25 \mathrm{~mm}$ at the whisker tip. We achieve $360^{\circ}$ active whisking by placing two such sensor modules diametrically opposite on a servo actuated rotary mount. When a whisker contacts an obstacle, a horizontal load stimulus is recorded as the corresponding magnet's deflection from the mean position.

\section{RESUlTS}

Fig. 1(B) shows three regions in the sensor's deflection characteristics from an experiment. In the $1^{\text {st }}$ region, the measured deflection gradually increases; in the $2^{\text {nd }}$, it becomes near-constant; in the $3^{\text {rd }}$, it restores to the mean

${ }^{1}$ Centre for Virtual Reality and Haptics, Indian Institute of Technology (IIT), Madras, India

${ }^{2}$ Information Technology and Electrical Engineering, University of Oulu, Finland. position with slight overshoot. The $1^{\text {st }}$ and $2^{\text {nd }}$ region shows the sensor's response before and after buckling, whereas the $3^{\text {rd }}$ region shows slip.
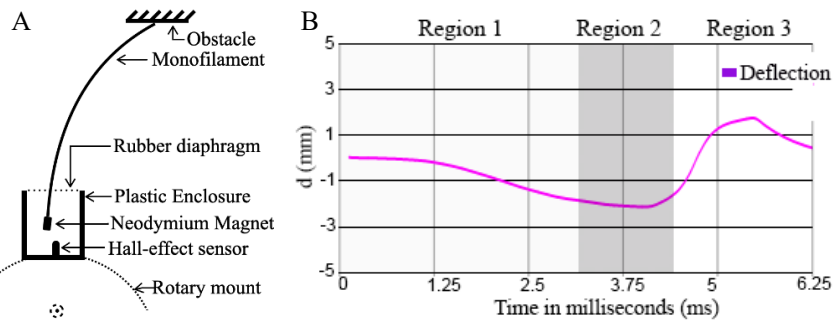

Figure 1. (A) Schematic of a single sensor module located on a rotary mount. (B) Sensor's response when a horizontal load is applied at the tip.

\section{DISCUSSION AND CONCLUSION}

This abstract presents a whisker-based tactile sensor for mobile robot navigation. The deflection characteristic has three distinct regions of operation that help detect buckling. The reproducible buckling load could enable the robot to determine a constant force stimulus of $10 \pm 1 \mathrm{~g}$, potentially helpful in detecting an obstacle's texture and compliance. The constant force approach is maybe more accurate and reliable for the application because [6], [7] found significant changes in biomechanics using the constant force method, whereas standard stress-strain extensometry could not. The sensitivity of the constant force technique needs to be studied. The design's advantages over the existing sensors are: (1) It can measure the whisker's tip deflection as well as a constant force stimulus. (2) The $360^{\circ}$ tactile view could help navigate narrow spaces. (3) Multiple sensor modules could be used in tactile arrays due to their small size.

Such sensors could help navigate in a vacuum, narrow or blind spaces. They potentially require lesser bandwidth and computation for obstacle perception compared to other modalities. The proposed design with a multimodal transducer could increase the range and reliability of measurements in unstructured environments [8]. In the future, we plan to test these hypotheses by deploying drones with such tactile sensors in underground burrows with varying temperatures, visibility, toxicity, and lighting.

\section{REFERENCES}

[1] R. A. Grant et al., Journal of Neurophysiology, vol. 101(2), 2009

[2] S. Kim et al., IEEE/RSJ International Conference on IROS, 2020

[3] D. Jung et al., IEEE/RSJ International Conference on IROS, 1996

[4] J. Bell-Krotoski et al., The Journal of hand surgery, vol. 12(1), 1987

[5] M. Manivannan et al., ICoRD'15, Research into Design Across Boundaries, 2015

[6] O. Richoz et al., PLoS One, vol. 9(8), 2014

[7] E. K. Kim et al., J Neuroeng Rehabil, vol. 9, 2012

[8] H. Wegiriya et al., IEEE International Conference on SMC, 2016 